\title{
Antitumor effect of manumycin on colorectal cancer cells by increasing the reactive oxygen species production and blocking PI3K-AKT pathway
}

This article was published in the following Dove Press journal:

OncoTargets and Therapy

24 May 2016

Number of times this article has been viewed

\author{
Jingyu Zhang' \\ Hua Jiang ${ }^{2}$ \\ Li Xie' \\ Jing $\mathrm{Hu}^{\prime}$ \\ $\mathrm{Li} \mathrm{Li}^{\prime}$ \\ Mi Yang' \\ Lei Cheng' \\ Baorui Liu' \\ Xiaoping Qian' \\ 'Department of the Comprehensive \\ Cancer Center, Affiliated Nanjing \\ Drum Tower Hospital, Nanjing Medical \\ University, ${ }^{2}$ Department of Oncology, \\ Affiliated Changzhou No 2 People's \\ Hospital, Nanjing Medical University, \\ Nanjing, People's Republic of China
}

Correspondence: Xiaoping Qian Department of the Comprehensive Cancer Center, Affiliated Nanjing Drum Tower Hospital, Nanjing Medical University, 32I Zhongshan Road, Nanjing 210008, People's Republic of China

Tel +86 I5। $05 \mid 32995$

Fax +862568182342

Email xiaopingqian@nju.edu.cn

\begin{abstract}
Manumycin is a natural, well-tolerated microbial metabolite and is regarded as a farnesyltransferase inhibitor. Some data suggest that manumycin inhibits proliferation of diverse cancer cells through various pathways. However, the antitumor effect of manumycin on colorectal cancer (CRC) remains unknown. In the present study, we investigated the antitumor effect of manumycin on CRC in vitro and in vivo. The results of cell viability assay revealed that the proliferation of the CRC cells was significantly inhibited by manumycin. Moreover, cell apoptosis induced by manumycin was also found in a time- and dose-dependent manner. Interestingly, treatment of the CRC cells with manumycin resulted in increased generation of reactive oxygen species. Subsequently, manumycin also decreased the phosphorylation of phosphatidylinositol 3-kinase (PI3K) and AKT, as well as the expression of caspase-9 and poly(ADP-ribose) polymerase (PARP) in a time-dependent manner. In addition, we found that $\mathrm{N}$-acetyl-L-cysteine (NAC) attenuated the effect of manumycin on the PI3K-AKT pathway, and wortmannin reduced the effect of manumycin on caspase-9 and PARP expression. More importantly, the anticancer effect of manumycin was also observed in established tumor xenografts. Taken together, these findings supported the potential application of manumycin against colorectal carcinoma.
\end{abstract}

Keywords: manumycin, colorectal cancer, PI3K-AKT pathway, ROS

\section{Introduction}

Colorectal cancer (CRC) is one of the most common malignant tumors of the gastrointestinal tract. Owing to the changes in adverse dietary habits, the incidence of CRC has increased gradually in the recent years. ${ }^{1}$ Meanwhile, $>25 \%$ of patients are diagnosed with metastatic disease. ${ }^{2}$ The metastatic CRC patients have a poor survival, and fluorouracil (5-FU) is a fundamental drug for chemotherapy of metastatic CRC. After irinotecan and subsequent oxaliplatin were approved, the overall survival of advanced CRC patients was found to be prolonged to $\sim 20$ months when treated with 5-FU combined with irinotecan or oxaliplatin. ${ }^{3,4}$ Targeted therapy, such as cetuximab, a monoclonal antibody that selectively inhibits the downstream activation of the epithelial growth factor receptor, has obtained great achievement in CRC treatment. ${ }^{5}$ Unfortunately, the use of the above standard therapeutic protocols results only in a moderate decline in mortality because not all CRCs are sensitive to this antiepidermal growth factor receptor agent. Therefore, more effort should be exerted to identify novel drug targets and therapeutics to prolong the survival time of CRC patients. 
Phosphatidylinositol 3-kinase (PI3K) was first discovered $>20$ years ago and is considered as one of the 15 driver mutations during CRC carcinogenesis. ${ }^{6}$ The effects of PI3K on tumor growth and progression are mediated by AKT, a downstream effector of PI3K. PI3K induces AKT activation via phosphorylation of key amino acid residues, including Thr-308 and Ser-473. Fully phosphorylated AKT activates a multitude of downstream targets, including the mTOR complex 1, BAD, caspase-9, and TSC $1 .{ }^{7}$ It has been demonstrated that the activation of caspase- 9 can lead to the activation of downstream caspases and apoptosis. ${ }^{8,9}$ Mutations in PI3K have been observed in 10\%-20\% of patients with CRC. ${ }^{10,11}$ Approximately 15\% of patients who showed resistance to cetuximab-based chemotherapy harbored a PIK3CA mutation. ${ }^{12}$ Many clinical trials are currently assessing novel drugs that interfere with signaling from components of the PI3K pathway. However, development of secondary resistance, unanticipated feedback effects, and pathway cross talk have challenged the efforts to design therapeutically effective compounds. ${ }^{13}$ Thus, drugs inhibiting different critical nodes within the PI3K pathway with less toxicity are urgently needed.

Manumycin, a natural product derived from Streptomyces parvulus that acts as a potent and selective farnesyltransferase inhibitor, was identified as an effective tumoricide against several cancers, including hepatocellular carcinoma, prostate cancer, and anaplastic thyroid carcinoma. ${ }^{14}$ It was revealed by histological examination that there was no apparent pathological change in the liver, kidney, and heart after manumycin treatment, indicating that it has the low-toxicity property of the agent. ${ }^{15}$ However, the detailed mechanisms of its actions are incompletely understood. A recent study reported that manumycin-induced apoptosis involved a cascade that began with a large reactive oxygen species (ROS) induction followed by dephosphorylation of MEK and AKT. ${ }^{16}$ On the other hand, the tumors containing phospho-stable MEK and AKT exhibited either a delayed response to manumycin-induced apoptosis or no response to apoptosis in the extended time. Moreover, manumycininduced autophagy was reported to be caused by elevated ROS. ${ }^{17}$ Additionally, in human CRC, manumycin may induce DNA damage via generation of ROS in HCT116 cells in vitro. ${ }^{18}$ Meanwhile, manumycin suppressed aberrant crypt foci formation in the azoxymethane-induced colorectal carcinogenesis model. ${ }^{19}$ When it comes to collaborative optimization, interestingly, combination of chemotherapeutic paclitaxel and manumycin could synergistically kill anaplastic thyroid carcinoma cells. ${ }^{20}$ Therefore, we hypothesized that manumycin could induce apoptosis associated with the ROS production in the human CRC cells.

\section{Materials and methods Cell culture}

The human CRC cell lines SW480 and Caco-2, obtained from the Cell Bank of the Chinese Academy of Sciences (Shanghai, People's Republic of China), were grown in Roswell Park Memorial Institute-1640 medium supplemented with 10\% fetal bovine serum and incubated in humidified $5 \% \mathrm{CO}_{2}$ at $37^{\circ} \mathrm{C}$. This study was conducted under a protocol approved by the institutional review board at Nanjing Medical University.

\section{Reagents}

Manumycin, dimethyl sulfoxide (DMSO; tissue culture grade), and 3-(4,5-dimethylthiazol-2-yl)-2,5-diphenyletrazolium bromide (MTT) were purchased from Sigma-Aldrich Co. (St Louis, MO, USA). Roswell Park Memorial Institute1640 medium was purchased from Thermo Fisher Scientific (Waltham, MA, USA). Fetal bovine serum was purchased from HyClone Laboratories, Inc. (Logan, UT, USA). Reactive Oxygen Species Assay Kit, $N$-acetyl-L-cysteine (NAC), and wortmannin were purchased from Beyotime Institute of Biotechnology (Haimen, Jiangsu, People's Republic of China). Trifluoroacetate salt hydrate was purchased from Cayman Chemical Company (Ann Arbor, MI, USA). Anti-PI3K antibody, anti-p-PI3K antibody, anti-AKT antibody, anti-p-AKT antibody, anti-caspase-9 antibody, and anti-poly(ADP-ribose) polymerase (PARP) antibody were purchased from Cell Signaling Technology (Danvers, MA, USA). Anti-proliferating cell nuclear antigen (PCNA) antibody was purchased from BD Biosciences (San Jose, CA, USA). $\beta$-Actin mouse monoclonal antibody, goat anti-rabbit secondary antibody, and horseradish peroxidase secondary antibody were purchased from Santa Cruz Biotechnology Inc. (Dallas, TX, USA). Annexin-V-FLUOS Staining Kit and In Situ Cell Death Detection Kit, POD, were purchased from Hoffman-La Roche Ltd. (Basel, Switzerland). Manumycin, being lipophilic was dissolved in DSMO. The stock solution was diluted with the final concentration of DSMO in a culture medium not exceeding $0.1 \%$ (volume/volume $[\mathrm{v} / \mathrm{v}]$ ).

\section{Cell proliferation assay}

The MTT assay was done to investigate the effect of manumycin on the growth of SW480 and Caco-2 cells. Logarithmic phase cells $\left(5 \times 10^{3}\right.$ cells/well $)$ were seeded in 96 -well tissue culture plates in $5 \% \mathrm{CO}_{2}$ at $37^{\circ} \mathrm{C}$. After culture of 24 hours for cell attachment, the medium was replaced with medium containing different concentrations 
of manumycin for the indicated time periods. In all, $20 \mu \mathrm{L}$ MTT dye $(5 \mathrm{mg} / \mathrm{mL})$ was added to each cell, and the plates were incubated at $37^{\circ} \mathrm{C}$ for another 4 hours. Then the medium was removed, and DMSO $(150 \mu \mathrm{L})$ was added. At last, the plates were shocked at a low volatility for 15 minutes in the darkness, and absorbance of each well at a wavelength of $490 \mathrm{~nm}$ was determined using a microplate reader. A decrease in absorbance was considered as a loss of cell viability. Each experiment was repeated for three times. The optical density was recorded as $\mathrm{OD}$, and the inhibition rate (IR\%) was calculated using the following equation:

$$
\operatorname{IR}(\%)=\frac{1-\text { Mean OD value in treatment group }}{\text { Mean OD value in control group }} \times 100 \% \text { (1) }
$$

\section{Apoptosis analysis}

The Annexin-V-FLUOS Staining Kit was used to measure the apoptosis cells. According to the manufacturer's instructions, cells were seeded in six-well plates at $2 \times 10^{5} /$ well and treated with different reagents for the indicated time periods. The cells were harvested, washed with cold phosphate-buffered saline (PBS) for three times, and resuspended in $400 \mu \mathrm{L}$ of bing buffer, subsequently $8 \mu \mathrm{L}$ Annexin-V-FITC solution, and propidium iodide was added to these cells. The cells were then incubated in the darkness for 30 minutes at $37^{\circ} \mathrm{C}$ followed by the analysis of the apoptosis cells by flow cytometry.

\section{Intracellular ROS level}

The Reactive Oxygen Species Assay Kit was used to study intracellular ROS. Cells were seeded in six-well plates at $2 \times 10^{5} /$ well and treated with different reagents for the indicated time periods. The medium was removed and replaced with the serum-free medium containing $10 \mu \mathrm{M}$ dichlorodihydro-fluorescein diacetate. After 20 minutes of incubation at $37^{\circ} \mathrm{C}$ in the darkness, the cells were washed with PBS for three times, and then fluorescent intensity was measured by the flow cytometer with excitation and emission wavelengths of $488 \mathrm{~nm}$ and $525 \mathrm{~nm}$, respectively.

\section{Western blot}

After various treatments, the culture medium and cells floating in the culture medium were removed. The cells that remained attached to the wells were rinsed with PBS for three times and lyzed in radioimmunoprecipitation assay buffer supplemented with a mixture of protease and phosphatase inhibitors, and the total protein was extracted from the cells. The protein concentration was determined by the bicinchoninic acid protein assay. The cell lysate was separated on $10 \%$ sodium dodecyl sulfate-polyacrylamide gel (SDS-PAGE). Equal amounts of total protein extracts were separated on a $10 \%$ SDS-PAGE and transferred onto a polyvinylidene fluoride membrane. Membranes were blocked with $10 \%$ fat-free milk in TBST for 2 hours and then incubated with the following primary antibodies at $4^{\circ} \mathrm{C}$ overnight: anti-PI3K antibody (diluted at 1:1,000), anti-p-PI3K antibody (diluted at 1:1,000), anti-AKT antibody (diluted at 1:1,000), anti-p-AKT antibody (diluted at 1:1,000), anticaspase-9 antibody (diluted at 1:1,000), and anti-PARP antibody (diluted at 1:1,000), followed by incubation with HRP secondary antibody (diluted at 1:3,000) for 1 hour. Protein bands were detected using the chemiluminescent HRP substrate. The blot was incubated for 5 minutes and then exposed to a suitable X-ray film for $\sim 30$ seconds. Bands were identified and quantified using AlphaEaseFC software.

\section{Inhibition of tumor growth by manumycin in vivo}

The activity of manumycin to inhibit tumor growth in vivo was evaluated in the xenograft nude mouse mode. Male athymic BALB/c mice ( 8 weeks old, 18-20 g) were purchased from Nanjing University Animals Laboratory (Nanjing, Jiangsu, People's Republic of China) and feed with enough food and water. The animals and their care was conducted in conformity with $3 \mathrm{R}$ principles of animal experimentation and approved by Animal Care and Use Ethic Committee of the Affiliated Nanjing Drum Tower Hospital, Nanjing Medical University. Since similar results in SW480 and Caco-2 cells in vitro were obtained, only SW480 cells $\left(1 \times 10^{6}\right)$ in $0.1 \mathrm{~mL}$ of culture medium were injected subcutaneously into the right scapular region of nude mice to evaluate the activity of manumycin in vivo. When the bearing tumors were $\sim 100 \mathrm{~mm}^{3}$ in size, mice were randomly assigned to the three groups. Either control solution $(0.1 \%$ DMSO in a tissue culture medium) or manumycin (low: $2.5 \mathrm{mg} / \mathrm{kg}$ and high: $5 \mathrm{mg} / \mathrm{kg})$ was administered by intraperitoneal injection $(\mathrm{n}=5$ each group) at a 2-day interval for 3 weeks. Tumor size was measured every 3 days using the formula $A \times B^{2} \times 0.5236$, where $A$ is length and $B$ is width; all were measured in millimeters. All mice were sacrificed on day 22, and tissue specimens were weighed and fixed in formalin for paraffin embedding.

\section{Determination of tumor cell proliferation}

Immunohistochemical PCNA staining of tumor tissues were used to evaluate proliferation activity of tumor cells. Briefly, paraffin-embedded tissues were deparaffinized and rehydrated prior to antibody addition. Anti-PCNA antibody was used at a dilution of 1:1,000. Goat anti-rabbit secondary antibody was used at a dilution of 1:200. To quantify 
PCNA expression, the PCNA labeling index was calculated as number of PCNA positive cells/total number of cells counted under $\times 200$ magnification in three randomly selected areas in each tissue section.

\section{Terminal deoxynucleotidyl transferase dUTP nick-end labeling detection of apoptotic tumor cells}

Terminal deoxynucleotidyl transferase dUTP nick-end labeling (TUNEL) staining was performed using the In Situ Cell Death Detection Kit, POD, following the manufacturer's recommended protocol. In brief, the formalin-fixed and paraffin-embedded tissues were cut into $5 \mu \mathrm{m}$ thick sections. Subsequently, the slides were incubated with the TUNEL reaction mixture containing terminal deoxynucleotidyl transferase enzyme and fluorescein-dUTP. After removing unbound enzyme conjugate, we visualized the POD retained cells in the immune complex using a substrate reaction. We quantified the percentage of apoptotic cells using Image J software. Under the light microscope at $\times 200$ magnification, we randomly chose three different visual areas within the same regions of each section. The positive rates of apoptotic cells were calculated as the density of apoptotic cells (apoptosis index).

\section{Statistical analysis}

We analyzed the comparisons between the control and treatment groups using one-factor analysis of variance and expressed them as mean \pm SD. The differences between two groups were further analyzed using Student-Newman-Keuls test. All data were analyzed with the Statistical Package for
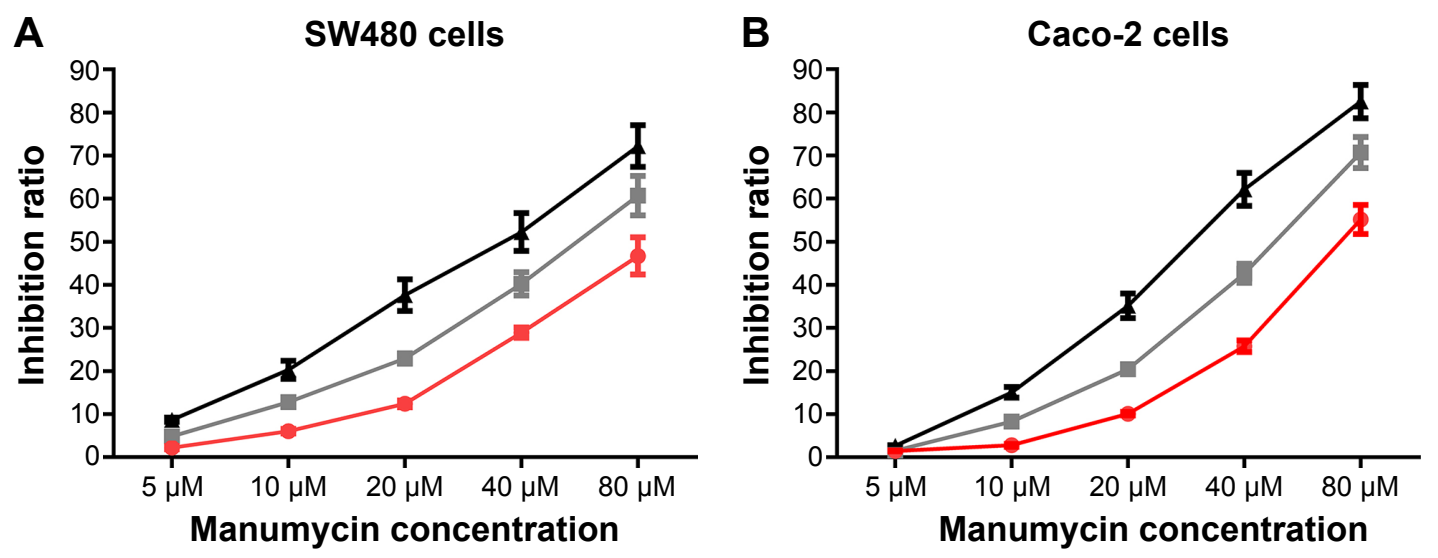

\section{Manumycin induces apoptosis in human CRC cells}

We conducted apoptotic analysis to determine whether manumycin induces apoptosis in the CRC cells. As shown in Figure 3, manumycin decreased the percentage of cell apoptosis in a dose- and time-dependent manner in two

$48 \mathrm{~h}=24 \mathrm{~h}=-12 \mathrm{~h}$

Figure I Manumycin inhibits proliferation of CRC cells.

Notes: (A) SW480 and (B) Caco-2 cells were treated with 0-80 $\mu$ M manumycin for 0-48 h. Cell viability was determined by MTT assay. Inhibiting rate was plotted against the manumycin concentration. The error bars represent the $95 \% \mathrm{Cls}$ of three independent experiments.

Abbreviations: CRC, colorectal cancer; h, hours; MTT, 3-(4,5-dimethylthiazol-2-yl)-2,5-diphenyletrazolium bromide; Cl, confidential interval. 

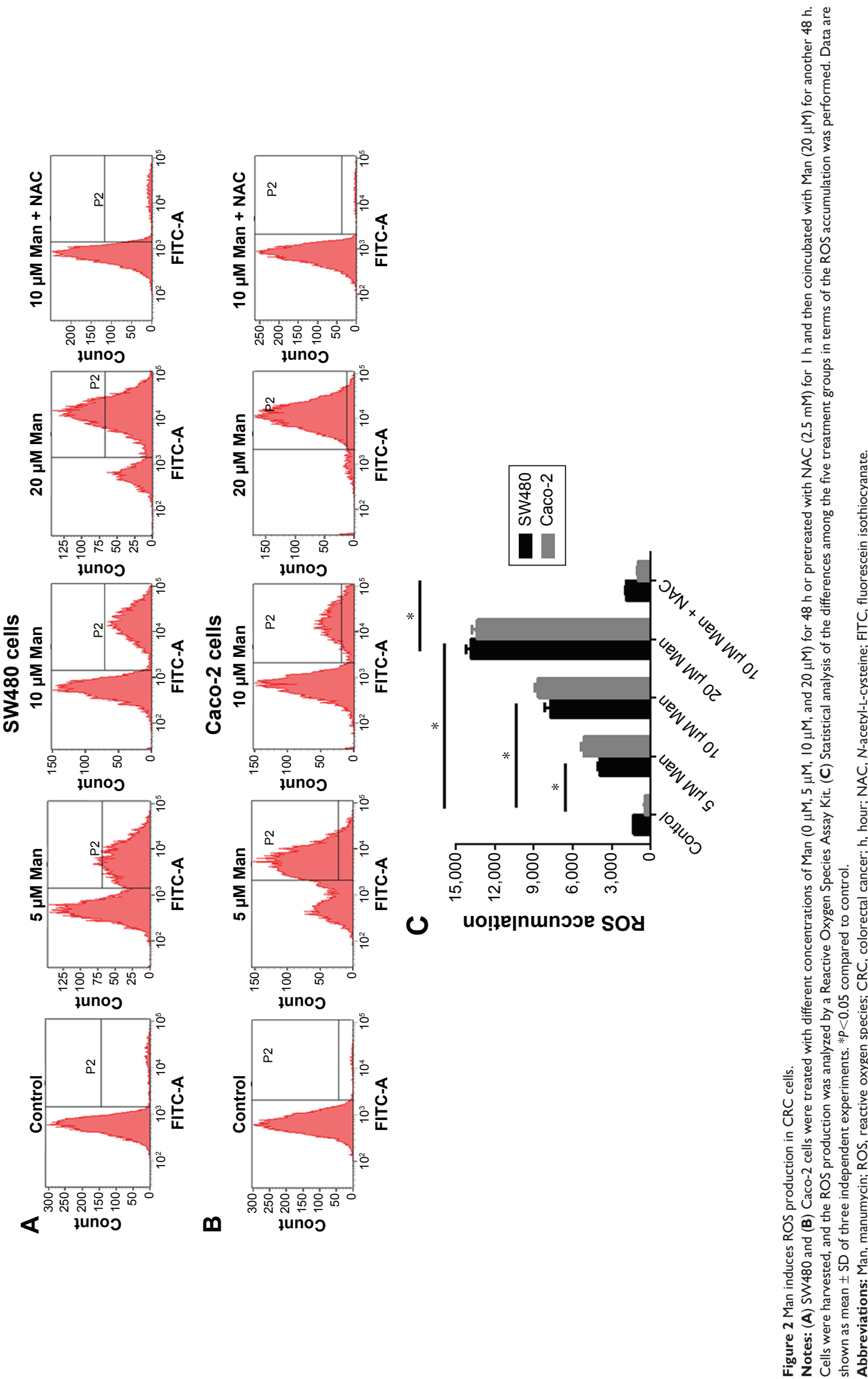
cell lines (Figure 3A, B, D, and E). Of note, when cells were treated with manumycin and NAC, the decrease in the apoptotic cells was weakened (Figure 3C and F). These results suggested that the apoptotic effect of manumycin was associated with the ROS production.

\section{Manumycin inhibits the PI3K-AKT pathway in human CRC cells}

To identify whether the PI3K-AKT pathway was involved in manumycin-induced apoptosis, we tested expression of relevant proteins using immunoblot analysis. In two cell lines, manumycin treatment reduced expression of $\mathrm{p}-\mathrm{PI} 3 \mathrm{~K}$ and $\mathrm{p}$-AKT in a time-dependent manner (Figure 4A and B). There was a similar trend in the expression of PI3K and AKT (Figure 4A and B). Pretreatment of cells with a PI3K inhibitor wortmannin for 1 hour decreased the activation of AKT phosphorylation (Figure 4C and D), while pretreatment of cells with NAC weakened the PI3K and AKT phosphorylation to a smaller extent (Figure 4E and F), indicating ROS production was a upstream of the PI3K-AKT pathway.
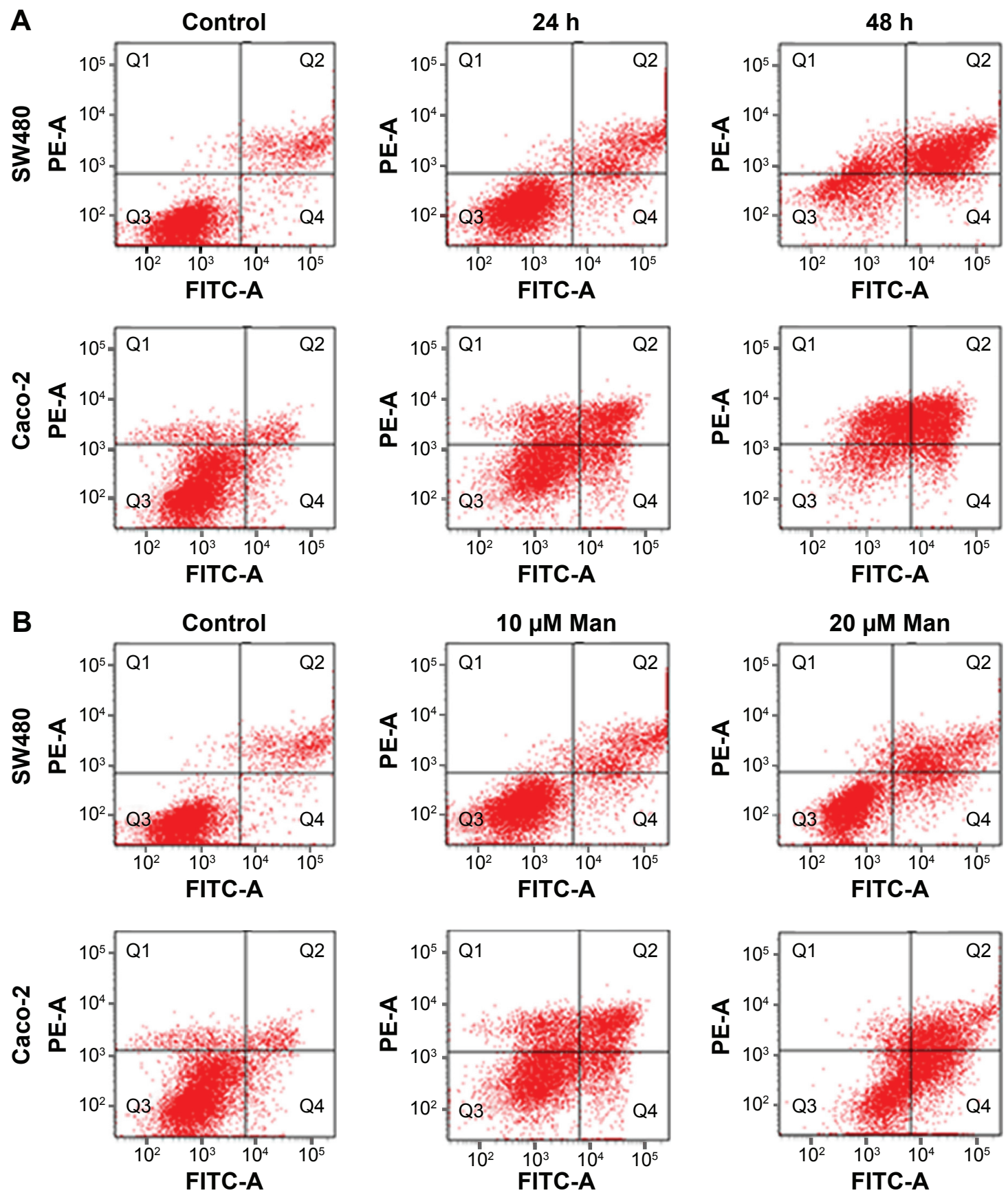

Figure 3 (Continued) 

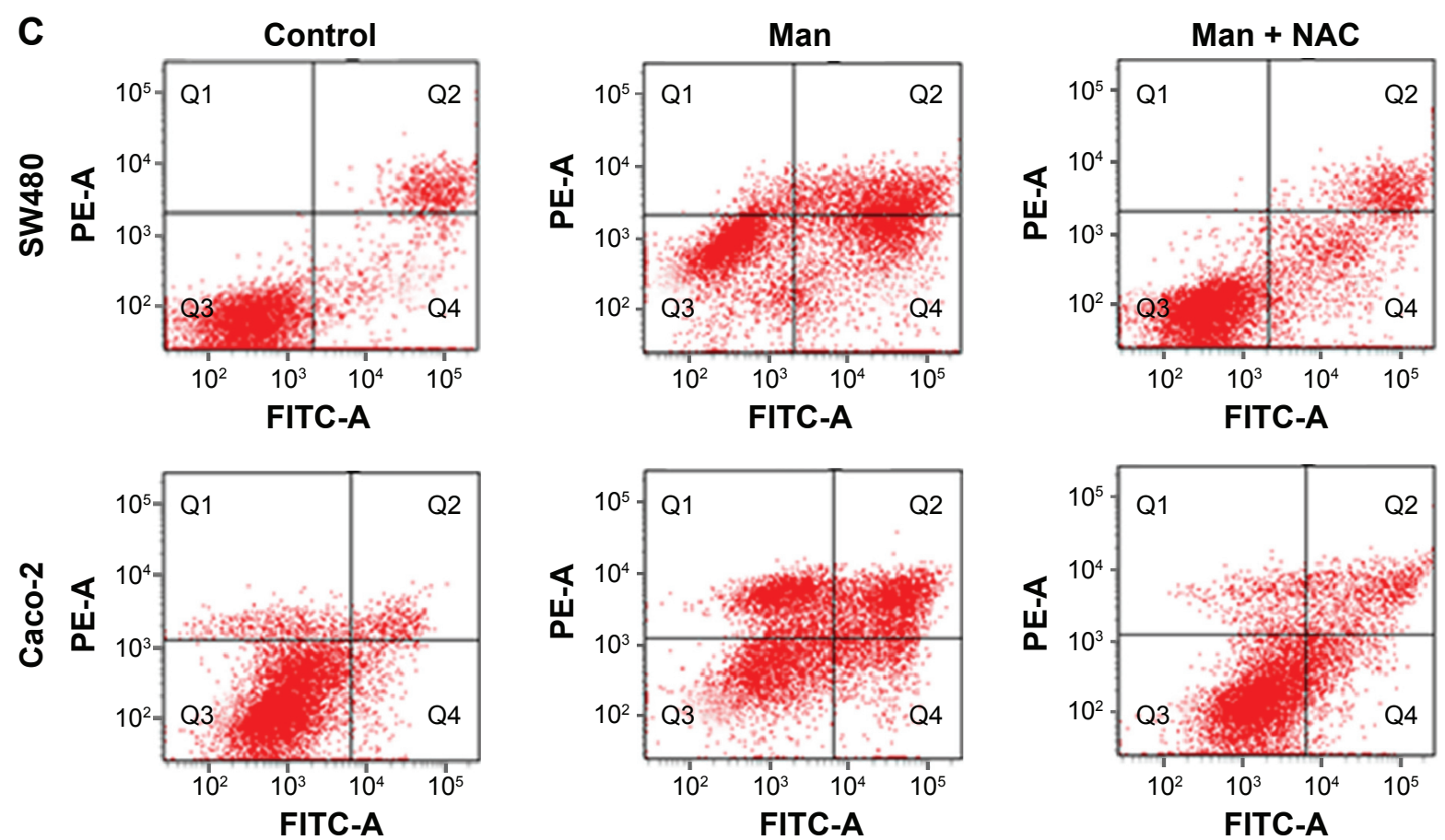

FITC-A
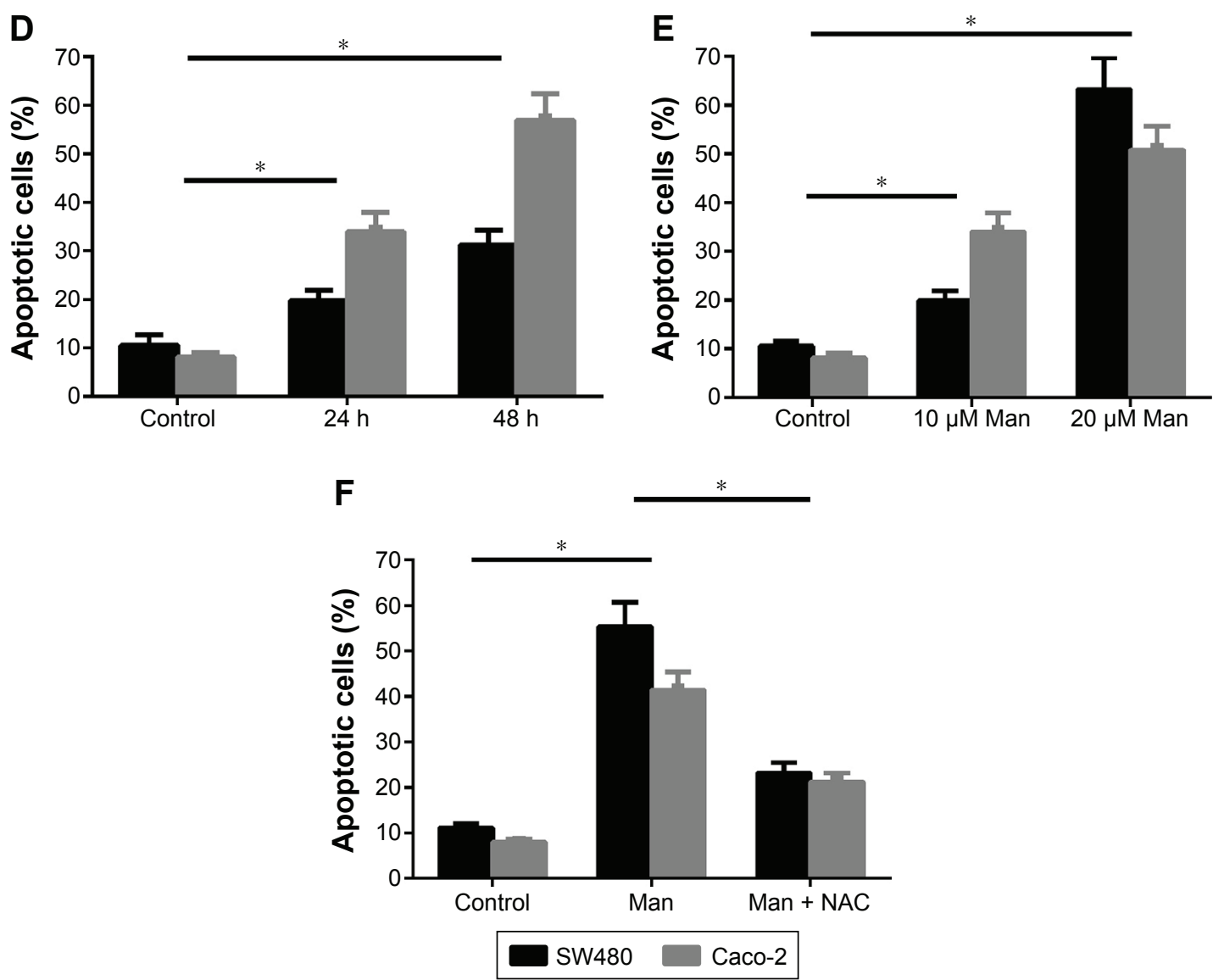

Figure 3 Man induces apoptosis in CRC cells.

Notes: Cells were treated with different concentrations and different courses. Cells were stained with Annexin-V/propidium iodide, and apoptosis was determined by flow cytometry. (A) Cells were treated with $10 \mu \mathrm{M}$ Man for different times. (B) Cells were treated with different concentrations of Man for 24 h. (C) Cells were pretreated with or without $2.5 \mathrm{mM} \mathrm{NAC}$ for I h and then coincubated with $20 \mu \mathrm{M}$ Man for $24 \mathrm{~h}$. (D-F) Statistical analysis of the differences among the three treatment groups in terms of apoptotic cells in terms of relative protein expression in $(\mathbf{A}-\mathbf{C})$ was performed. Data are shown as mean \pm SD of three independent experiments. $* P<0.05$ compared to control.

Abbreviations: Man, manumycin; CRC, colorectal cancer; h, hour; NAC, N-acetyl-L-cysteine; FITC, fluorescein isothiocyanate; SD, standard deviation. 
A

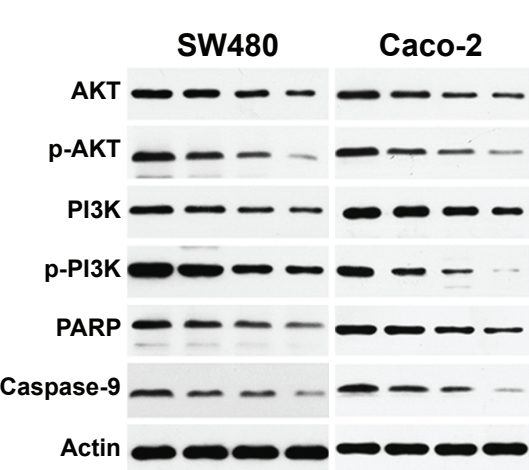

O h 12 h 24 h 48 h 0 h 12 h24 h48 h
B

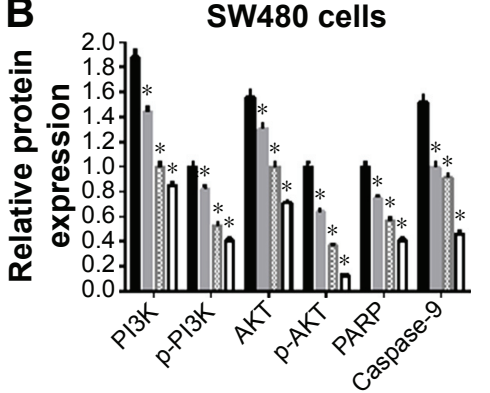

- Control $12 \mathrm{~h}$ ․ㅣㅆ $24 \mathrm{~h} \square 48 \mathrm{~h}$

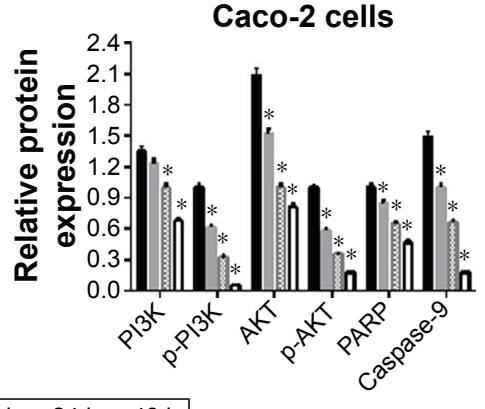

C SW480 Caco-2
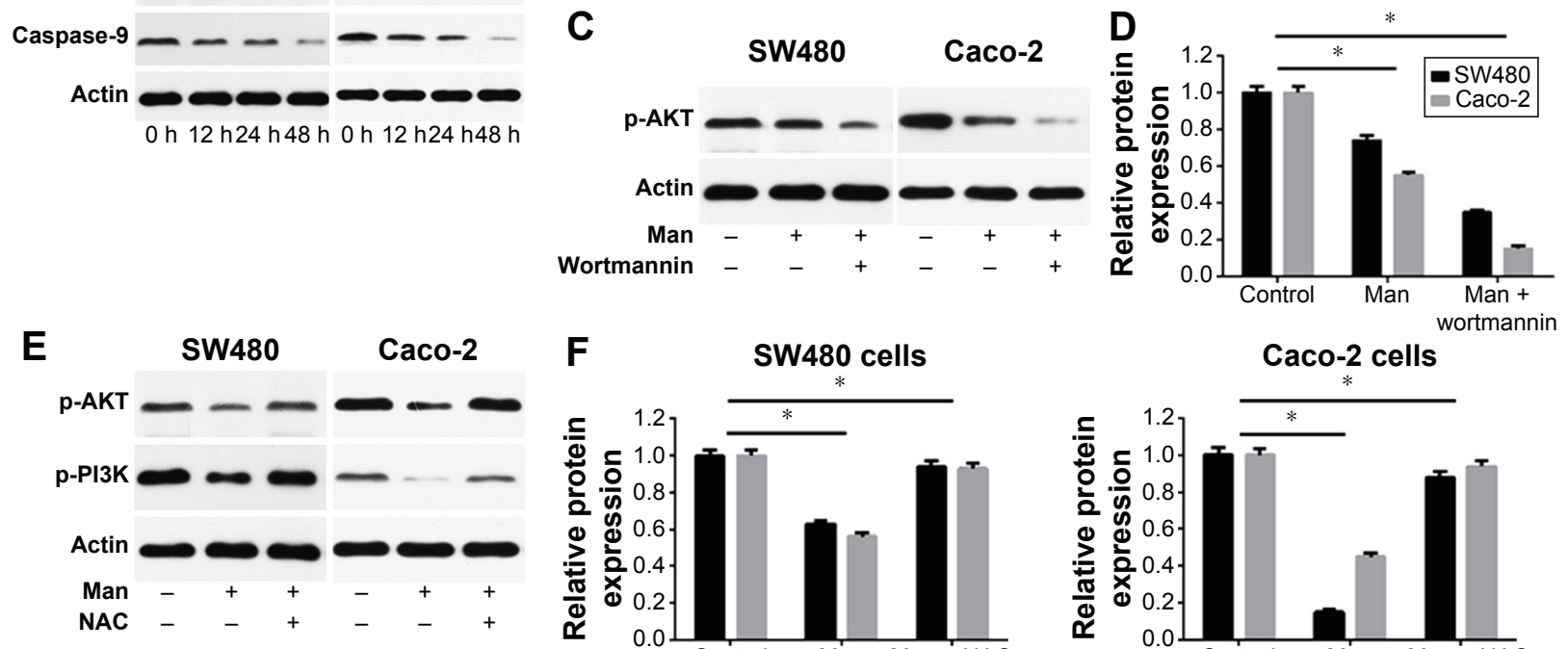

F

SW480 cells
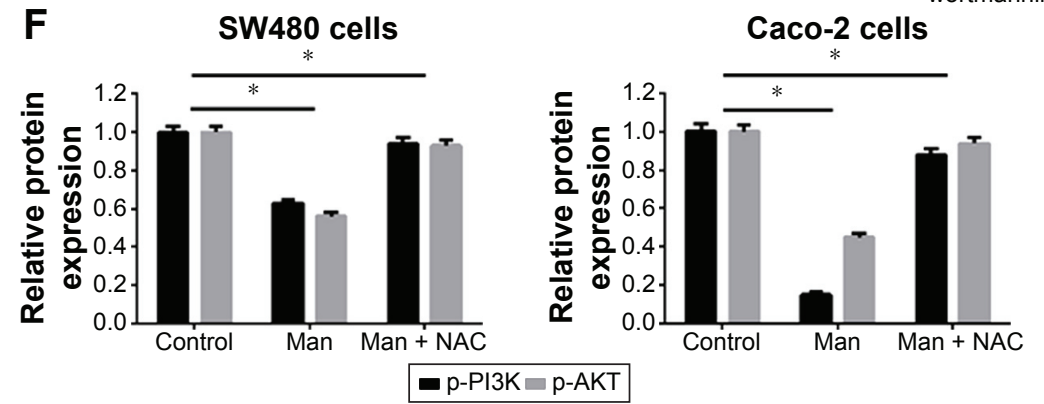

G
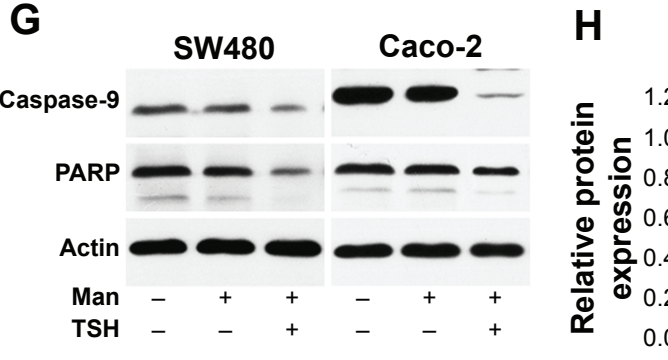

H SW480 cells
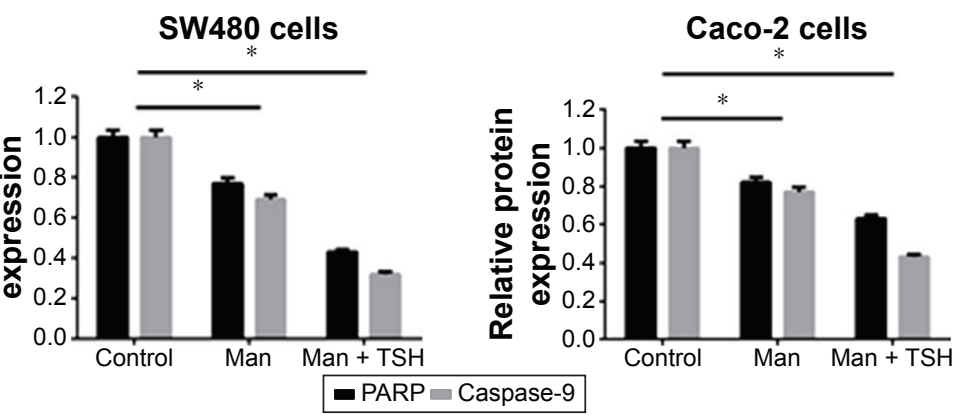

Figure 4 Manumycin inhibits PI3K-AKT pathway.

Notes: Cells were treated with Man and lyzed. The relevant proteins in the PI3K-AKT pathway were analyzed by Western blot. (A) Cells were treated for various times $(0 \mathrm{~h}, 12 \mathrm{~h}, 24 \mathrm{~h}$, and $48 \mathrm{~h})$ with $10 \mu \mathrm{M}$ Man. (B) Statistical analysis of the differences among the four treatment groups in terms of relative protein expression in (A) was performed. (C) Cells were pretreated with or without wortmannin (I5 $\mu \mathrm{M})$ for I h and then coincubated with $10 \mu \mathrm{M}$ Man for another $48 \mathrm{~h}$. (D) Statistical analysis of the differences among the three treatment groups in terms of relative protein expression in (C) was performed. (E) Cells were pretreated with or without NAC (2.5 mM) for $\mathrm{I} \mathrm{h}$ and then coincubated with $10 \mu \mathrm{M}$ Man for another $48 \mathrm{~h}$. (F) Statistical analysis of the differences among the three treatment groups in terms of relative protein expression in (E) was performed. (G) Cells were pretreated with or without TSH (I5 $\mu$ M) for I h and then coincubated with $10 \mu \mathrm{M}$ Man for another $48 \mathrm{~h}$. (H) Statistical analysis of the differences among the three treatment groups in terms of relative protein expression in (G) was performed. Data are shown as mean \pm SD of three independent experiments. $* P<0.05$ compared to control.

Abbreviations: PI3K, phosphatidylinositol 3-kinase; Man, manumycin; h, hour; NAC, N-acetyl-L-cysteine; TSH, trifluoacetate salt hydrate; PARP, poly(ADP-ribose) polymerase; SD, standard deviation.

\section{Manumycin decreased expression of caspase-9 and PARP in human CRC cells}

The caspase family includes important proteins that perform apoptosis. Caspase-9 is the major initiator of the intrinsic apoptotic death pathway, which is activated by mitochondrial perturbation. Caspase- 9 then activates caspase- 3 and other caspases, subsequently inactivating the DNA repair enzyme PARP. Our results showed that manumycin decreased expression of caspase- 9 and PARP in a time-dependent manner (Figure 4A and B). Furthermore, the cells were 
pretreated with an AKT inhibitor, trifluoroacetate salt hydrate, and the treatment with manumycin led to a further decrease in caspase-9 and PARP (Figure 4G and H). Overall, these data showed that manumycin influenced the level of PARP and capase-9 proteins by modulating the PI3KAKT pathway.

\section{Manumycin significantly inhibits the growth of CRC in vivo}

The ability of manumycin as a tumor suppressor was also assessed in the tumor xenograft model. As shown in Figure 5, administration of manumycin by intraperitoneal injection inhibited SW480-derived tumor growth in a dose-dependent manner (Figure 5A). Consistent with tumor volumes, the average tumor weight at 22 days was $0.804 \pm 0.059 \mathrm{~g}$ and $0.42 \pm 0.03 \mathrm{~g}$ at the dose of $2.5 \mathrm{mg} / \mathrm{kg}$ and $5.0 \mathrm{mg} / \mathrm{kg}$, respectively, being obviously reduced compared with the control group (Figure 5B, $P<0.05$ ). Cell proliferation marker PCNA was further examined in situ in tumor samples from the three groups. Manumycin-treated groups had reduced expression of PCNA in comparison with the control group (Figure 5C). Meanwhile, tumor tissue was subjected to TUNEL assay for the determination of apoptotic index. Cell nuclei were stained with nigger brown, which indicated apoptosis and
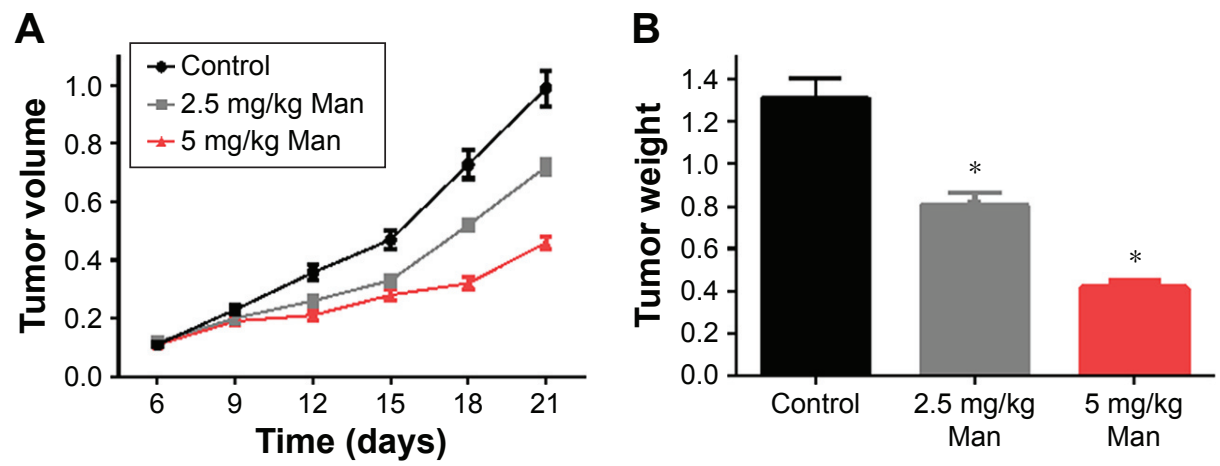

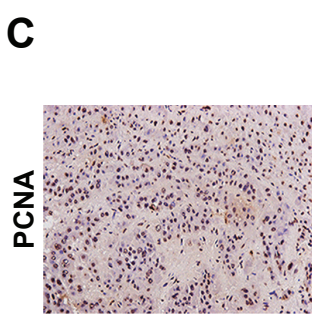

Control

D

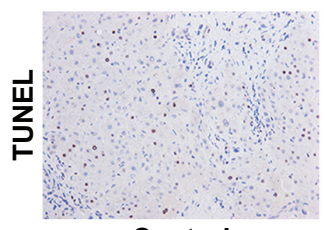

Control

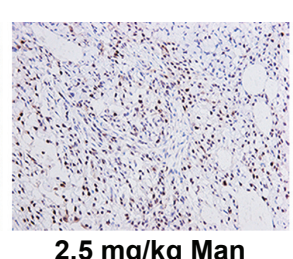

$2.5 \mathrm{mg} / \mathrm{kg}$ Man

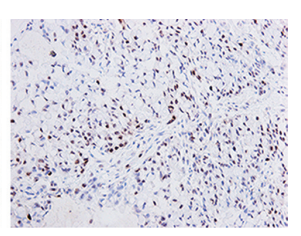

$5 \mathrm{mg} / \mathrm{kg}$ Man
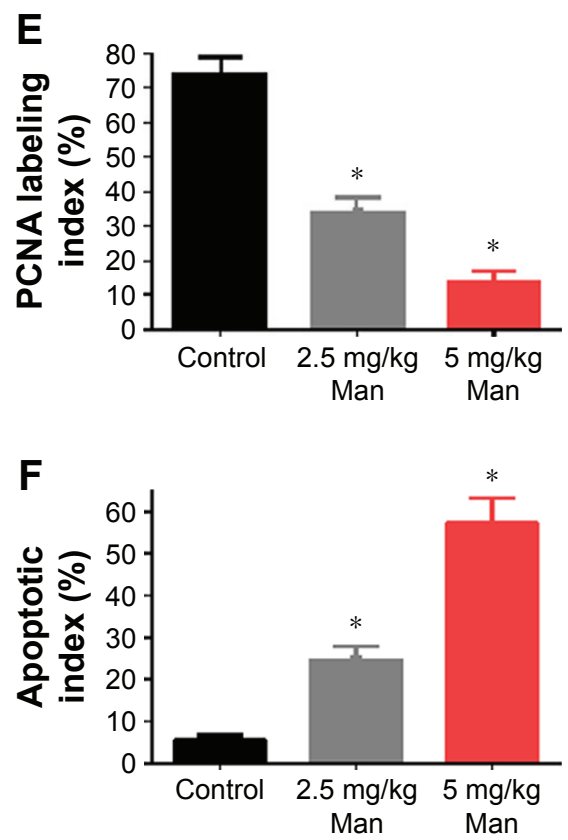

Figure 5 Man significantly inhibits the growth of CRC in vivo.

Notes: SW480 cells were injected sc into the right scapular region of nude mice to evaluate the activity of manumycin in vivo. When the bearing tumors were $\sim 100$ mm ${ }^{3}$, mice were randomly assigned to the three groups. Either control solution ( $0.1 \%$ DMSO in a tissue culture medium) or manumycin (low: $2.5 \mathrm{mg} / \mathrm{kg}$ and high: $5 \mathrm{mg} / \mathrm{kg})$ was administered by ip injection ( $\mathrm{n}=5$ each group) at a 2-day interval for 3 weeks. All mice were sacrificed on day 22. (A) Mean tumor volumes measured every 3 days by caliper measurement of two perpendicular diameters of the tumors. (B) Tumor weights of tumor tissues from different groups at 22 days after injection. (C) Proliferation analysis of tumor tissues by PCNA immunohistochemistry staining. (D) Apoptosis analysis of tumor tissues by TUNEL assay. (E and F) Statistical analysis of the differences among the three treatment groups in terms of PCNA labeling index and apoptotic index. Data are shown as mean \pm SD of three independent experiments. $* P<0.05$ compared to control.

Abbreviations: Man, manumycin; CRC, colorectal cancer; sc, subcutaneously; DMSO, dimethyl sulfoxide; ip, intraperitoneal; PCNA, proliferating cell nuclear antigen; TUNEL, terminal deoxynucleotidyl transferase dUTP nick-end labeling. 
were recorded as TUNEL-positive nuclei. The manumycintreated group had a significantly higher apoptosis index of tumor cells when compared with the control group (Figure 4D). Moreover, we found no loss in body weights in group treated with manumycin (data not shown). Taken together, manumycin was demonstrated as an efficient and low-toxicity agent against tumors in vivo.

\section{Discussion}

Our data provided evidence that manumycin inhibited proliferation and induced apoptosis in the CRC cells. We also demonstrated that manumycin inhibited PI3K-AKT activation and led to ROS accumulation and caspase-9 activation in the CRC cells.

The cellular redox homeostasis is maintained by a tight balance between the rates of ROS production and the ability of the various antioxidant defense systems to modulate redox status. It is well known that the cellular redox status has an important role in varieties of cellular process, including cell proliferation and apoptosis..$^{21,22}$ Our results showed the anticancer activity of manumycin on colorectal cells was concomitant with the elevated level of intracellular ROS. Moreover, given that antioxidants NAC inhibited accumulation of manumycinstimulated ROS and attenuated the apoptotic effect on the CRC cells induced by manumycin, the antitumor effect of manumycin might be mediated by ROS generation.

$\mathrm{PI} 3 \mathrm{~K}$ is an intracellular lipid kinase that plays an important role in cell function and cancer development. Reportedly, PI3K pathway is activated by four principal types of sensors, including the receptor tyrosine kinases, the cytokine- and G-proteincoupled receptors, and the integrins. ${ }^{7,23-25}$ Upon appropriate binding, these sensors, in conjunction with their cofactors, activate downstream kinases in the PI3K family. PI3K activation leads to the recruitment of $\mathrm{AKT}$ and its subsequent phosphorylation by PDK1 and mTORC2 by phosphorylating phosphatidylinositol 4,5-biphosphate to phosphatidylinositol 3,4,5-triphosphate. Phosphorylated AKT exerts signals leading to cell growth and differentiation and angiogenesis and prevents apoptosis by activating a multitude of downstream targets, including caspase-9. We found that manumycin inhibited the phosphorylation of PI3K and AKT. Moreover, the combination of the PI3K inhibitor and manumycin inhibits the phosphorylation of AKT in both CRC cell lines.

Apoptosis is tightly regulated by proapoptotic and antiapoptotic effector molecules and can be mediated by several different pathways in response to various intrinsic and extrinsic signals. ${ }^{26}$ Caspase- 9 is the major initiator of the intrinsic apoptotic death pathway, which results from mitochondrial perturbation. ${ }^{27}$ Caspase- 9 activates caspase- 3 and other effector caspases, which proceed to cleave and inactivate the DNA repair enzyme PARP. Hence, PARP cleavage is used as a classic readout for upstream effector caspase activity. Our results indicated that manumycin induced the apoptosis of CRC cell lines and decreased the expression of caspase-9 and PARP, which may be associated with caspase-9 and PARP cleavage. Moreover, the AKT inhibitor significantly decreased the expression of caspase-9 and PARP induced by manumycin. These data confirmed that apoptosis induced by manumycin required capase- 9 activation and AKT was an upstream to the caspase-9 activation.

Some studies have demonstrated that wortmannin, a PI3K-AKT inhibitor, considerably inhibits the translocation of nicotinamide adenine dinucleotide phosphate oxidase subunits and reduces ROS production, indicating that PI3K signaling might be involved in this progress. ${ }^{28,29}$ Actually, the catalytic activity of PTEN is also modulated by ROS, and the cellular PTEN phosphatase activity is inhibited by the oxidative stress. ${ }^{30}$ Considering the role of PTEN in modulating the PI3K-AKT pathway, there is a feedback loop between ROS and the PI3K-AKT pathway. At the same time, due to massive ROS production in cancer cells, the effective redox adaptation mechanisms would be coped with noxious environment. ${ }^{31}$ Cancer cells are more vulnerable to oxidative stress caused by exogenous ROS-generating agents. Owing to differential redox states between normal and cancer cells, manumycin might benefit colorectal tumor patients. Our data found that manumycin induced ROS production and subsequently inhibited activation of PI3K and AKT, resulting in cancer cell apoptosis. However, the exact mechanism of ROS production induced by manumycin is not fully understood and is currently under investigation in our laboratory.

\section{Conclusion}

In our study, we found that manumycin inhibited proliferation and induced apoptosis on SW480 and Caco-2 cells through modulation of the PI3K-AKT pathway. ROS production played an important role in this progress. Moreover, the anticancer effect of manumycin was further confirmed in an in vivo study of CRC. Our findings provide a preclinical evidence for the potential use of manumycin in the treatment of CRC in the future. However, more work is needed to establish the exact mechanism of manumycin activity in the CRC cells.

\section{Acknowledgments}

The authors thank all the people who helped in this study. This work partly was supported by Grant 2011-WS-005 from 
the Six Talents Peak Foundation of Jiangsu Province, Grant ZKX13022 from the Department of Health key projects of Nanjing City, and Grant 201503013 from Science and Technology Development Program of Nanjing city, Jiangsu Province, People's Republic of China.

\section{Disclosure}

The authors report no conflicts of interest in this work.

\section{References}

1. Kamangar F, Dores GM, Anderson WF. Patterns of cancer incidence, mortality, and prevalence across five continents: defining priorities to reduce cancer disparities in different geographic regions of the world. J Clin Oncol. 2006;24(14):2137-2150.

2. Ciombor KK, Wu C, Goldberg RM. Recent therapeutic advances in the treatment of colorectal cancer. Annu Rev Med. 2015;66:83-95.

3. Saltz LB, Cox JV, Blanke C, et al. Irinotecan plus fluorouracil and leucovorin for metastatic colorectal cancer. Irinotecan Study Group. N Engl J Med. 2000;343(13):905-914.

4. de Gramont A, Figer A, Seymour M, et al. Leucovorin and fluorouracil with or without oxaliplatin as first-line treatment in advanced colorectal cancer. J Clin Oncol. 2000;18(16):2938-2947.

5. Van Cutsem E, Kohne CH, Hitre E, et al. Cetuximab and chemotherapy as initial treatment for metastatic colorectal cancer. NEngl J Med. 2009; 360(14):1408-1417.

6. Sjoblom T, Jones S, Wood LD, et al. The consensus coding sequences of human breast and colorectal cancers. Science. 2006;314(5797): 268-274.

7. Danielsen SA, Eide PW, Nesbakken A, Guren T, Leithe E, Lothe RA. Portrait of the PI3K/AKT pathway in colorectal cancer. Biochim Biophys Acta. 2015;1855(1):104-121.

8. Gurumurthy S, Vasudevan KM, Rangnekar VM. Regulation of apoptosis in prostate cancer. Cancer Metastasis Rev. 2001;20(3-4):225-243.

9. Fesik SW. Promoting apoptosis as a strategy for cancer drug discovery. Nat Rev Cancer. 2005;5(11):876-885.

10. Frattini M, Signoroni S, Pilotti S, et al. Phosphatase protein homologue to tensin expression and phosphatidylinositol-3 phosphate kinase mutations in colorectal cancer. Cancer Res. 2005;65(23):11227.

11. Velho S, Oliveira C, Ferreira A, et al. The prevalence of PIK3CA mutations in gastric and colon cancer. Eur J Cancer. 2005;41(11):1649-1654.

12. De Roock W, Claes B, Bernasconi D, et al. Effects of KRAS, BRAF, NRAS, and PIK3CA mutations on the efficacy of cetuximab plus chemotherapy in chemotherapy-refractory metastatic colorectal cancer: a retrospective consortium analysis. Lancet Oncol. 2010;11(8):753-762.

13. Yu M, Grady WM. Therapeutic targeting of the phosphatidylinositol 3-kinase signaling pathway: novel targeted therapies and advances in the treatment of colorectal cancer. Therap Adv Gastroenterol. 2012;5(5): 319-337.

14. Li JG, She MR, Lu CY, et al. Manumycin induces apoptosis in prostate cancer cells. Onco Targets Ther. 2014;7:771-777.

15. Ito $T$, Kawata $S$, Tamura $S$, et al. Suppression of human pancreatic cancer growth in BALB/c nude mice by manumycin, a farnesyl:protein transferase inhibitor. Jpn J Cancer Res. 1996;87(2):113-116.

OncoTargets and Therapy

\section{Publish your work in this journal}

OncoTargets and Therapy is an international, peer-reviewed, open access journal focusing on the pathological basis of all cancers, potential targets for therapy and treatment protocols employed to improve the management of cancer patients. The journal also focuses on the impact of management programs and new therapeutic agents and protocols on
16. Sears KT, Daino H, Carey GB. Reactive oxygen species-dependent destruction of MEK and Akt in Manumycin stimulated death of lymphoid tumor and myeloma cell lines. Int J Cancer. 2008;122(7):1496-1505.

17. Pan J, Song E, Cheng C, Lee MH, Yeung SC. Farnesyltransferase inhibitors-induced autophagy: alternative mechanisms? Autophagy. 2009;5(1):129-131.

18. Pan J, She M, Xu ZX, Sun L, Yeung SC. Farnesyltransferase inhibitors induce DNA damage via reactive oxygen species in human cancer cells. Cancer Res. 2005;65(9):3671-3681.

19. Tsuda M, Okamoto K, Muguruma N, et al. Suppressive effect of RAS inhibitor manumycin A on aberrant crypt foci formation in the azoxymethane-induced rat colorectal carcinogenesis model. J Gastroenterol Hepatol. 2013;28(10):1616-1623.

20. Pan J, Yeung SC. Recent advances in understanding the antineoplastic mechanisms of farnesyltransferase inhibitors. Cancer Res. 2005;65(20): 9109-9112.

21. Scatena R. Mitochondria and cancer: a growing role in apoptosis, cancer cell metabolism and dedifferentiation. Adv Exp Med Biol. 2012;942: 287-308.

22. Nakanishi A, Wada Y, Kitagishi Y, Matsuda S. Link between PI3K/ AKT/PTEN pathway and NOX proteinin diseases. Aging Dis. 2014;5(3): 203-211.

23. Chang F, Lee JT, Navolanic PM, et al. Involvement of PI3K/Akt pathway in cell cycle progression, apoptosis, and neoplastic transformation: a target for cancer chemotherapy. Leukemia. 2003;17(3):590-603.

24. Su CC, Lin YP, Cheng YJ, et al. Phosphatidylinositol 3-kinase/Akt activation by integrin-tumor matrix interaction suppresses Fas-mediated apoptosis in T cells. J Immunol. 2007;179(7):4589-4597.

25. Papadatos-Pastos D, Rabbie R, Ross P, Sarker D. The role of the PI3K pathway in colorectal cancer. Crit Rev Oncol Hematol. 2015;94(1): 18-30.

26. Chipuk JE, Fisher JC, Dillon CP, Kriwacki RW, Kuwana T, Green DR. Mechanism of apoptosis induction by inhibition of the antiapoptotic BCL-2 proteins. Proc Natl Acad Sci U S A. 2008;105(51): 20327-20332.

27. Heck DE, Kagan VE, Shvedova AA, Laskin JD. An epigrammatic (abridged) recounting of the myriad tales of astonishing deeds and dire consequences pertaining to nitric oxide and reactive oxygen species in mitochondria with an ancillary missive concerning the origins of apoptosis. Toxicology. 2005;208(2):259-271.

28. Ren Z, Raucci FJ Jr, Browe DM, Baumgarten CM. Regulation of swelling-activated $\mathrm{Cl}(-)$ current by angiotensin II signalling and NADPH oxidase in rabbit ventricle. Cardiovasc Res. 2008;77(1):73-80.

29. Ngkelo A, Meja K, Yeadon M, Adcock I, Kirkham PA. LPS induced inflammatory responses in human peripheral blood mononuclear cells is mediated through NOX4 and Gialpha dependent PI-3kinase signalling. J Inflamm (Lond). 2012;9(1):1.

30. Barbieri SS, Ruggiero L, Tremoli E, Weksler BB. Suppressing PTEN activity by tobacco smoke plus interleukin-1beta modulates dissociation of VE-cadherin/beta-catenin complexes in endothelium. Arterioscler Thromb Vasc Biol. 2008;28(4):732-738.

31. Li ZY, Yang Y, Ming M, Liu B. Mitochondrial ROS generation for regulation of autophagic pathways in cancer. Biochem Biophys Res Commun. 2011;414(1):5-8.

patient perspectives such as quality of life, adherence and satisfaction. The manuscript management system is completely online and includes a very quick and fair peer-review system, which is all easy to use. Visit http://www.dovepress.com/testimonials.php to read real quotes from published authors. 